\title{
BMJ Open Protocol for a feasibility study investigating the UCalgary's Cannabis Café: education and harm reduction initiative for postsecondary students
}

\author{
Joel Mader (D , ${ }^{1}$ Jacqueline M Smith, ${ }^{1}$ Jennifer Smith, ${ }^{1}$ Darren Robert Christensen ${ }^{2}$
}

To cite: Mader J, Smith JM, Smith J, et al. Protocol for a feasibility study investigating the UCalgary's Cannabis Café: education and harm reduction initiative for postsecondary students. BMJ Open 2020;10:e032651. doi:10.1136/ bmjopen-2019-032651

- Prepublication history and additional material for this paper are available online. To view these files, please visit the journal online (http://dx.doi. org/10.1136/bmjopen-2019032651).

Received 28 June 2019 Revised 23 December 2019 Accepted 23 January 2020

\section{Check for updates}

(c) Author(s) (or their employer(s)) 2020. Re-use permitted under CC BY-NC. No commercial re-use. See rights and permissions. Published by BMJ.

${ }^{1}$ Faculty of Nursing, University of Calgary, Calgary, Alberta, Canada

${ }^{2}$ Health Sciences, University of Lethbridge, Lethbridge, Alberta, Canada

Correspondence to

Joel Mader;

joel.mader@ucalgary.ca

\section{ABSTRACT}

Introduction High rates of cannabis consumption among emerging adults in Canada represent an important public health issue. As part of the legalisation of cannabis, health objectives were established by the Government of Canada including reducing risky patterns of consumption and cannabis related harm among vulnerable populations. Despite these ambitions, few evidenced based education programmes have been evaluated in the literature. The aim of this study is to describe and evaluate the acceptability of a novel harm reduction and education initiative titled, UCalgary's Cannabis Café. The Cannabis Café incorporates components shown to be effective in reducing risky substance consumption on campuses and substance related stigma. An important objective of the Café is the dissemination of methods to reduce risk in the form of Canada's Lower-Risk Cannabis Use Guidelines.

Methods and analysis The study will take the form of a non-experimental, observational cohort design, where participants will be asked to complete four surveys (baseline, immediate follow-up, 1 month and 3 months). The primary outcomes of the study will be the feasibility of the initiative including acceptability and implementation. Secondary outcomes include knowledge uptake of methods to reduce risk of cannabis related harm, descriptive cannabis norms and changes in cannabis consumption.

Ethics and dissemination The study was approved by the University of Calgary Conjoint Health Research Ethics Board (\#REB18-1364). The investigators will develop a guideline outlining the Cannabis Café to assist in the replication of this initiative at other locations and publish the results from the study in a peer-reviewed manuscript.

\section{INTRODUCTION}

Canadian youth (15-24-year olds) are consuming cannabis at higher rates than youth in most other nations of the world. ${ }^{1}$ Emerging adults (18-24-year olds), the demographic to which most university students belong, have historically demonstrated the highest rates of cannabis use when compared with other age groups. ${ }^{2}$ Society's changing beliefs about cannabis use and its shifting legal status appear to be impacting young people's

\section{Strengths and limitations of this study}

- The Cannabis Café represents a brief psychoeducation initiative enhanced by normative feedback and goal setting that aims to promote uptake of the Canada's Lower-Risk Cannabis Use Guidelines to reduce cannabis related harm among postsecondary students.

- This study will explore a novel approach to promoting lower-risk cannabis use in the naturalistic setting of undergraduate classrooms.

- Casual assumptions will be limited, given the nonexperimental, observational design of the study and the lack of a control group.

- Generalisability of the findings will be limited and cannot be applied to populations outside of postsecondary contexts.

decisions around cannabis use, including declining perceptions that it is harmful. ${ }^{3}$ Preliminary evidence from USA suggests that cannabis use among university students may increase in states where it has been legalised for recreational consumption. ${ }^{4}$ Early evidence from the National Cannabis Survey revealed an increase in past 3-month cannabis use among Canadians 15 years and older in the first quarter following legalisation. ${ }^{5}$ However, the rates of cannabis use among Canadians under the age of 25 years remained relatively unchanged. Nevertheless, rates of use among youth and emerging adults continue to remain higher when compared with Canadians 25 years and older (30\% vs $16 \%$, respectively). ${ }^{5}$ High rates of cannabis consumption among adolescents and emerging adults reflect an important public health issue given the well-documented concern that regular cannabis use among this population is associated with an increased risk of negative health outcomes. ${ }^{6}$ For example, previous research has shown that approximately $10 \%$ of those who experiment with cannabis will develop 
a cannabis use disorder (CUD). ${ }^{7}$ The literature also demonstrates a significant relationship between cannabis use and psychotic related disorders among those with a genetic predisposition. ${ }^{8}$

In addition, there is correlational evidence that cannabis consumption may be associated with changes in brain structure and function, and that chronic, heavy use may result in impaired cognitive functioning in areas such as memory and learning. ${ }^{9}$ Consistent with these findings, cannabis use among university students has also been associated with compromised academic performance and poorer scholastic outcomes. ${ }^{10} 11$ Despite growing evidence highlighting the risks associated with cannabis use, research on cannabis perceptions has shown that youth often lack clarity on how cannabis affects the brain and body, ${ }^{12}$ which are important factors to understand risks and harms associated with use.

In light of the recent legalisation of recreational cannabis, the relatively high rates of use among emerging adults, and increasing evidence associated with negative outcomes among youth, there is growing recognition that further public education efforts are needed ${ }^{9}$ and that university campuses represent an important context for addressing cannabis related harm. ${ }^{13}$ However, few educational initiatives targeting cannabis use among emerging adults have been described or evaluated in the literature. Of the brief interventions that have been studied, the most common outcome used to measure efficacy was frequency of consumption. ${ }^{14}$ Although reductions in frequency may represent one way to reduce harm, harm reduction is much broader, and is characterised by methods to mitigate negative health outcomes despite continued use. ${ }^{15}$

\section{Lower-Risk Cannabis Use Guidelines (LRCUG)}

A resource with growing prominence in the literature is the LRCUG authored by Fischer and colleagues. ${ }^{16}$ These guidelines represent an evidenced based harm reduction framework including 10 recommendations to reduce risk of cannabis related harm. The recommendations include, but are not limited to, abstaining from cannabis, limiting frequency of use, using lower-risk methods of administration (eg, edibles vs burnable products) and avoiding deep inhalation (eg, holding breath after inhaling burnt cannabis to maximise effect).

Although LRCUG appear promising, their uptake and efficacy for reducing harm have not been well studied. For example, Manthey's ${ }^{15}$ research explored an earlier version of the LRCUG among a Canadian sample of 277 adult cannabis consumers, where half of the sample was randomly assigned to receive the recommendations delivered as part of an online survey. Intentions to change cannabis behaviours including patterns of consumption (eg, avoiding deep inhalation) and levels of use (eg, frequency) served as dependent variables. Those participants exposed to the LRCUG demonstrated greater intentions to change patterns of consumption when compared with the control group, while intention to change levels of use did not differ between groups. A significant limitation of this study was the cross-sectional design, and as such, it is unclear if intentions influenced behaviours. Another study completed by Fischer and colleagues ${ }^{17}$ explored if the LRCUG could be incorporated as part of two brief interventions (oral and written versions) using a sample of 134 Canadian postsecondary students who were heavy cannabis users. Participants in the treatment group demonstrated a significant decrease in deep inhalation, risky forms of ingestion and cannabis impaired driving at follow-up when compared with the control group.

Although early findings from these studies show promise that the LRCUG may serve as an important resource for mitigating harm, the uptake of the guidelines remains a relatively understudied area. Furthermore, it is unclear what methods are most effective in disseminating and promoting uptake of the LRCUG on campuses. The authors are not aware of any educational harm reduction initiatives that have used these guidelines to promote lower-risk consumption of cannabis among postsecondary students. This is notable, given the wide dissemination of the LRCUG in Canada.

\section{Brief interventions for cannabis}

A recent meta-analysis of 26 studies of brief interventions for cannabis use among emerging adults demonstrated that these initiatives yielded minimal changes with respects to reductions in frequency of cannabis consumption or consequences. ${ }^{14}$ There was, however, evidence to show that these brief interventions reduced CUD symptoms and promoted higher rates of abstinence at shortterm follow-up. Halladay and colleagues ${ }^{14}$ note that many of these studies may have been under powered and although effects from this interventions may be small, such initiatives may represent an important part of a continuum of care for addressing cannabis related harm. While there have been investigations of brief interventions, limited research has been done investigating what components make-up effective initiatives for preventing cannabis related harm.

In contrast, most interventions targeting addictive behaviours among postsecondary students have focused on alcohol use, with less attention being given to other substances including cannabis. ${ }^{18}$ Although alcohol and cannabis represent vastly different substances, differing in effects, patterns of consumption and prevalence, many insights can be gained from the literature on initiatives targeting alcohol related harm when developing and delivering initiatives targeting cannabis use among postsecondary students. For example, in a systematic review and meta-analysis of 41 studies with over 24000 firstyear university students the following were identified as components to be most effective for changing students' alcohol practices through brief interventions ${ }^{19}$ :

- Providing strategies to moderate use.

- Personal goal setting to moderate use.

- Norm correction (eg, descriptive norms) or personalised feedback, where peer and personal use is 
contrasted with norms from respective demographic groups

Descriptive norms refer to an individual's perceptions regarding the consumption of substances by others (eg, asking individuals to estimate the percentage of students who consumed cannabis in the past month ${ }^{20}$ ). It has been demonstrated that Canadian postsecondary students consistently overestimate the frequency of cannabis consumption among the student body. ${ }^{21}$ Some studies have demonstrated that descriptive norms are significantly associated with frequency of cannabis consumption and negative cannabis consequences ${ }^{2223}$ while others have failed to find such an association. ${ }^{24}$ Although there is some discrepancy in these early findings, Pearson and colleagues $^{23}$ argue that initiatives that correct norms may also lead to a reduction in cannabis related harm.

\section{Stigma}

Stigma is another factor that may contribute to cannabis related harm. Prior to legalisation of recreational cannabis, previous research has shown that recreational consumers reported hiding their use from family or co-workers for fear of social disapproval. ${ }^{25}$ Charlebois and colleagues (personal communication, 2019) study of 1051 Canadian adults (56\% of which reported having consumed cannabis in their lifetime) explored cannabis related stigma 6 months post legalisation of recreational cannabis. Roughly $19 \%$ reported not wanting to be seen buying cannabis from a government sponsored facility. Thirty-five per cent reported not wanting to work with someone who used cannabis regularly, and $24 \%$ reported not wanting co-workers to know if they used recreational cannabis. In addition, the recent 2019 Canadian Cannabis Survey ${ }^{26}$ which explored cannabis use and perceptions among a sample of 12000 Canadians found that approximately $23 \%$ of respondents indicated that despite the change in legal status, they were not more willing to publicly disclose cannabis consumption. These findings suggest that stigma related to cannabis use persists for some despite the change in legal status.

Bottorff and colleagues ${ }^{27}$ define two forms of stigma: external and internal. External stigma represents stigmatisation of others who have deviated from social norms, while internal stigma refers to negative beliefs held by an individual about themselves or a group that they belong to. Stigma related to cannabis use and substance use in general represents an important construct to be addressed, as it has been associated with adverse psychosocial outcomes. ${ }^{27-29}$ Of concern is stigma's effect on individuals who keep their drug use secretive, which can limit access to support for problematic use. ${ }^{30} 31$ Additionally, self-stigma can lead to lowered self-esteem, shame and decreased self-efficacy. ${ }^{32}$

Although we are not aware of any literature that has explored the effects of stigma related to recreational cannabis use, there is a portion of recreational consumers (although a minority), who continue to note reluctance in self-disclosing despite the change in legal status. ${ }^{26}$ It seems plausible that reluctance to divulge use may limit disclosures to healthcare providers. This concern is echoed by Wynn and colleagues ${ }^{, 33}$ recently published commentary. The authors argue that as with other legal substances such as tobacco, cannabis consumption will likely remain a stigmatised topic despite legalisation, especially among more intensive consumers and vulnerable populations such as youth. Failure to disclose such information to healthcare providers will likely limit the opportunity for patient education including the dissemination of information such as risk factors, potential harms and methods to lower risk. This in turn may lead to adverse outcomes or riskier use. In our review of the literature no brief cannabis interventions or education programmes targeted stigma. This may represent a missed opportunity given that these initiatives may be adapted to help mitigate the negative effects of stigma.

Several factors are effective at reducing stigma associated with mental health conditions including targeting prejudices and beliefs, and inviting direct contact between those with and without mental illness. ${ }^{28}$ In relation to substance use stigma, Brown ${ }^{28}$ argues that stigma reduction initiatives should be substance specific given that stigma varies among specific substances. Guided by Brown's suggestions, we have included methods in the Cannabis Café to challenge cannabis specific stigmatising beliefs by promoting discussion between users and non-users.

\section{Objectives}

The main objectives of the study are:

1. Describe a novel, in person, classroom-based education programme that is enhanced by components found to be effective in reducing substance related harm (ie, goal setting, normative feedback and methods to reduce harm) among university students.

2. Explore if components previously shown to be effective at reducing stigma relative to mental illness can be successfully incorporated into this initiative.

3. Determine the feasibility of this education programme among postsecondary students.

4. Determine if the Cannabis Café is effective at disseminating and promoting harm reduction as measured by adherence to the LRCUG. ${ }^{16}$

\section{METHODS AND ANALYSIS \\ Intervention}

The Cannabis Café (as overviewed in table 1 and described in detail in the online supplementary file) will integrate the following components:

1. Dissemination of evidenced based methods to reduce risky cannabis consumption via LRCUG.

2. Provide descriptive feedback to modify overestimations of cannabis consumption on campus, using prevalence data collected as part of a 2018 campus wide survey. ${ }^{13} 34$

3. Facilitate the application of information presented (eg, LRCUG) through an individualised activity where par- 


\begin{tabular}{|c|c|}
\hline Component & Description \\
\hline $\begin{array}{l}\text { Trivia ( 1 hour in } \\
\text { length) }\end{array}$ & $\begin{array}{l}\text { Facilitated using MentiMeter (an online } \\
\text { trivia platform where students are able } \\
\text { to answer questions anonymously via } \\
\text { their mobile or digital devices). } \\
\text { Used to disseminate information on } \\
\text { cannabis and normative feedback. } \\
\text { Facilitate discussions of information } \\
\text { following the review of each trivia } \\
\text { question. }\end{array}$ \\
\hline $\begin{array}{l}\text { World Café } \\
\text { ( 30 min in } \\
\text { length) }\end{array}$ & $\begin{array}{l}\text { Discussion among smaller groups led } \\
\text { by discussion leaders. } \\
\text { Round 1: addresses students' } \\
\text { perspectives toward LRCUG. } \\
\text { Round 2: seeks to challenge } \\
\text { stereotypes regarding cannabis use and } \\
\text { facilitate direct contact between those } \\
\text { with and without lived experience. } \\
\text { Following each round, discussions } \\
\text { are synthesised and major themes are } \\
\text { shared with the larger group. }\end{array}$ \\
\hline $\begin{array}{l}\text { Goal setting } \\
\text { activity ( } 5 \mathrm{~min} \\
\text { in length) }\end{array}$ & $\begin{array}{l}\text { As part of the immediate follow-up } \\
\text { survey, participants will be asked to } \\
\text { set a specific, measurable, achievable, } \\
\text { realistic and timely SMART goal to be } \\
\text { accomplished over the next } 30 \text { days } \\
\text { regarding their personal cannabis use } \\
\text { (users) or informing others' cannabis } \\
\text { use/maintaining abstinence (non-users). }\end{array}$ \\
\hline
\end{tabular}

total estimated time for the Cannabis Café is $95 \mathrm{~min}$. Please see online supplementary file 1 for detailed descriptions of trivia items (including respective answers, supporting resources and followup questions), World Café discussion points and the goal setting activity.

ticipants will be asked to set goals to be accomplished over the next 30 days following the Cannabis Café by considering ways to limit cannabis related harms (if using), or ideas to maintain abstinence (non-users), and/or consider ways in which they could share/disseminate the LRCUG to peers or family members.

4. Address internal and external stigma by challenging widely held prejudices and promoting direct contact through discussion between those who have and have not used cannabis.

As noted in table 1, we estimate the Cannabis Café will take $\sim 95 \mathrm{~min}$ for participants to complete. The trivia will be led by a group facilitator, who is a graduate level trained registered provisional psychologist with clinical experience working with substance using populations. Each topic of the World Café will be introduced by the group facilitator and will include reviewing each of the 10 recommendations described within the LRCUG. The group facilitator will also present each of the questions for the World Café discussion to all attendees via MentiMeter. Following the introduction of the World Café topics, undergraduate student discussion leaders, who will be trained in advance of the Cannabis Café (see discussion leader section for training protocol description) will facilitate smaller group discussions among attendees. Following each round of World Café, the group facilitator will ask discussion leaders to review themes that emerged from the smaller group discussions with all attendees.

\section{Patient and public involvement}

Two primary methods were used to gather student feedback: (1) the formation of a student advisory group during the initial development of the Cannabis Café and (2) gathering feedback collected from students during early (fall 2018) pilots of the Cannabis Café. Students' perspectives were used to establish the trivia format, the title of the initiative and the topics covered within the Cannabis Café.

\section{Study design}

The study is non-experimental and is an observational prospective design, where a cohort of participants will be followed for approximately 3 months. Outcomes will be measured at four time periods (baseline, immediate follow-up, 1-month follow-up and 3-month follow-up). All four surveys will be administered online using the Qualtrics platform. We estimate that each survey can be completed within $10 \mathrm{~min}$ or less. The baseline survey will ask participants for their email address so that responses can be matched and postintervention surveys can be sent. Participants will have up to 7 days to complete each survey and a reminder will be sent to participants 3-4 days after the initial invitation. Participants will be provided an honorarium after completing each survey including electronic $\$ 5$ gift cards for the first three surveys (baseline, immediate follow-up, 1-month follow-up, up to a total of $\$ 15$ ) and a $\$ 10$ gift card for the last survey (3-month follow-up). The incentive for the last survey was increased to promote full participation and reduce attrition.

\section{Participants}

Only those undergraduate students enrolled in a class on campus at UCalgary, whose instructor agreed to the Cannabis Café being delivered as course curriculum will be eligible for participation. All students enrolled in these classes will be eligible regardless of the severity of their cannabis use (or lack thereof). Given that the LRCUG is a harm reduction resource, we anticipate that all prospective and current cannabis consumers stand to benefit from the Cannabis Café. In particular, those who are more frequent or intensive users may stand to benefit the most by identifying ways to limit their risk by altering consumption patterns such as avoiding deep inhalation or avoiding synthetic products. Undergraduate classes will be targeted to ensure that the majority of eligible participants are under 25 years of age.

Given the limited research that has focused on harm reduction, normative correction was used to calculate sample size. Based on previous research ${ }^{20} 35$ a medium effect (Cohen's $f=0.265$ ) size was used yielding a minimum sample of $n=32$ past 30 -day users are necessary to capture 
effects. To achieve sufficient representation of students who report recent cannabis use, we are aiming to recruit up to a total of 500 participants. Based on national data collected from Canadian postsecondary samples, ${ }^{36}$ it is estimated that approximately $18 \%$ of participants will report past 30-day use, translating to an estimated sample of $n=90$ past 30 days cannabis users. We intentionally oversampled to ensure that we had a sufficient representation of cannabis consumers to offset attrition and to allow for exploration of secondary outcomes. Based on this sample size, we will be able to capture effects on secondary outcomes ranging between Cohen's $f=0.16$ and 0.265 .

\section{Recruitment}

Recruitment and data collection began in March 2019 and will continue until March 2020. Instructors teaching classes relevant to substance use and/or mental health will be targeted and invited to have the Cannabis Caféfacilitated by the research team as part of course curriculum. This will ensure continuity and relevancy of the Cannabis Café with course material. One week prior to delivering the initiative, the principle investigator, Dr Jacqueline Smith, will introduce the Cannabis Café and research project to students. Afterwards the course instructor/professor will send an anonymous link via email to each student inviting them to participate. Those who wish to participate will be asked to follow a link to informed consent and the baseline survey. Figure 1 is a flow diagram describing how participants progress through the study.

\section{Discussion leaders}

Discussion leaders trained by the research team will be present at each table to help facilitate discussion during the World Café. Training will occur through a mock Cannabis Café, where discussion leaders will become familiar with the content by taking the role of participants. The discussion leaders will also receive training on how to facilitate discussions during the World Café including the use of open-ended questions, ways to highlight important themes and protocols for supporting students who may become distressed. Protocols for handling distressed students will be able seek immediate support from the harm reduction specialist, a masters level trained social worker from the University student wellness centre who will be in attendance at each Café to debrief and provide appropriate referrals as needed. It is estimated that the training session will take approximately 4 hours. Discussion leaders will be recruited from undergraduate and graduate nursing students, and other relevant student groups on campus (eg, existing men's mental health group). Expectations and roles will be reviewed with discussion leaders prior to the commencement of each Cannabis Café. Four research staff including the principle investigator will be in attendance at each Cannabis Café to ensure protocols for the initiative are followed. Discussion leaders will also be debriefed by the group facilitator, principal investigator and research team following the conclusion of each Cannabis Café where difficulties or deviation from study protocols will be discussed.

\section{Feasibility and outcome measures}

The primary outcome of the study will be to measure the feasibility of the Cannabis Café. As described by Bowen and colleagues, ${ }^{37}$ feasibility studies are most appropriate when investigating novel initiatives where limited evidence exists. Of the eight areas outlined by Bowen and colleagues ${ }^{37}$ we determined acceptability and implementation to be most relevant. Beyond feasibility, the study will also explore several secondary outcomes including knowledge uptake among participants, changes in descriptive norms, stigma and harm reduction as operationalised by greater adherence to the LRCUG. Other information to be collected as part of the surveys include demographic information, questions related to personal use of cannabis and problematic use. Table 2 provides a visual representation of when each construct will be measured during the study.

\section{Demographic information}

Demographic information will be collected as part of the baseline survey which will include age, gender, sex and ethnicity.

\section{Feasibility}

Acceptability

Following Bowen and colleagues' ${ }^{37}$ description of acceptability, we will measure participants' reaction to the Cannabis Café including:

1. Was the Cannabis Café a good use of classroom time?

2. Should the Cannabis Café be included in next year's curriculum?

3. Would you recommend the Cannabis Café to a friend?

Participants will be asked to rate each component of the Cannabis Café (eg, the trivia and the World Café discussion) with respect to interest, enjoyment and if it was informative. Finally, participants will be asked if the topics addressed in the Cannabis Café were relevant and if the format of the Cannabis Café created a safe and nonjudgmental space to discuss personal experiences.

\section{Implementation}

Implementation addresses to what degree an initiative can be delivered as planned. ${ }^{37}$ Examples of implementation measures used in our study include, but are not limited to:

-Will instructors/professors express interest in hosting Cannabis Café during classroom hours?

- Will students demonstrate interest in becoming discussion leaders? How many of these students will attend the discussion leader training? How many trained discussion leaders will attend the Cannabis Cafe?

- How many participants will complete the goal setting activity post Cannabis Cafe?

- Of the students enrolled in each class, how many will consent to participate in the research? How many 


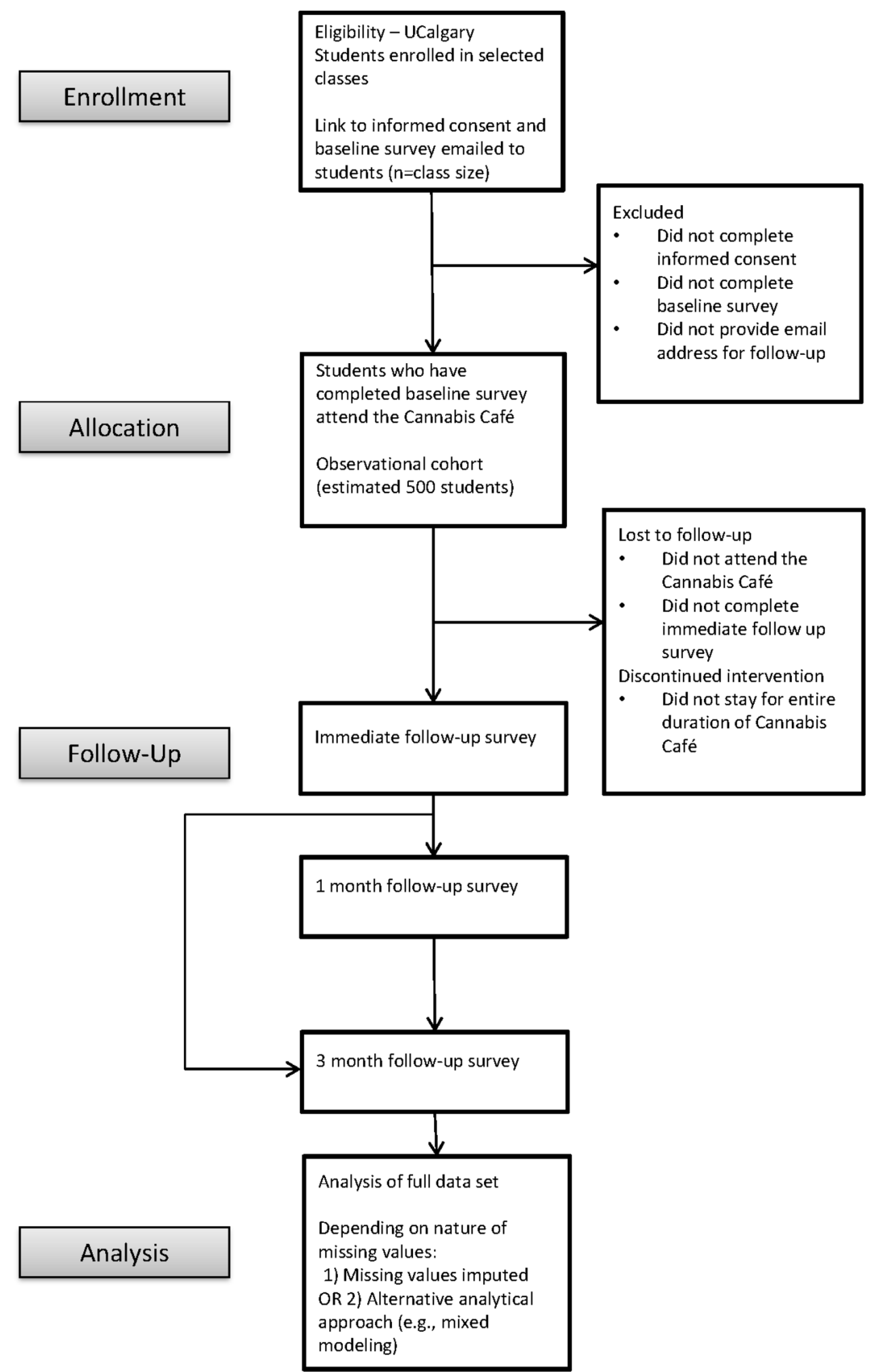

Figure 1 Consolidated Standards of Reporting Trials (CONSORT) diagram of how participants will progress through the study.

students from each course will attend the Cannabis Café during their scheduled class time?

\section{Descriptive norms}

Descriptive norms will be measured by asking participants to estimate the total number of days (within the last 30 days) that the average UCalgary student consumed cannabis.

\section{Cannabis use}

Participants will be asked if they have used cannabis in their lifetime (yes/no), if their use was authorised for medical purposes (yes/no) and if they primarily purchase through a legal retailer (yes/no). We will also estimate prevalence of CUD among the sample using the Cannabis Use Disorder Identification Test-Revised (CUDIT-R). ${ }^{38}$ 
Table 2 Measures administration schedule

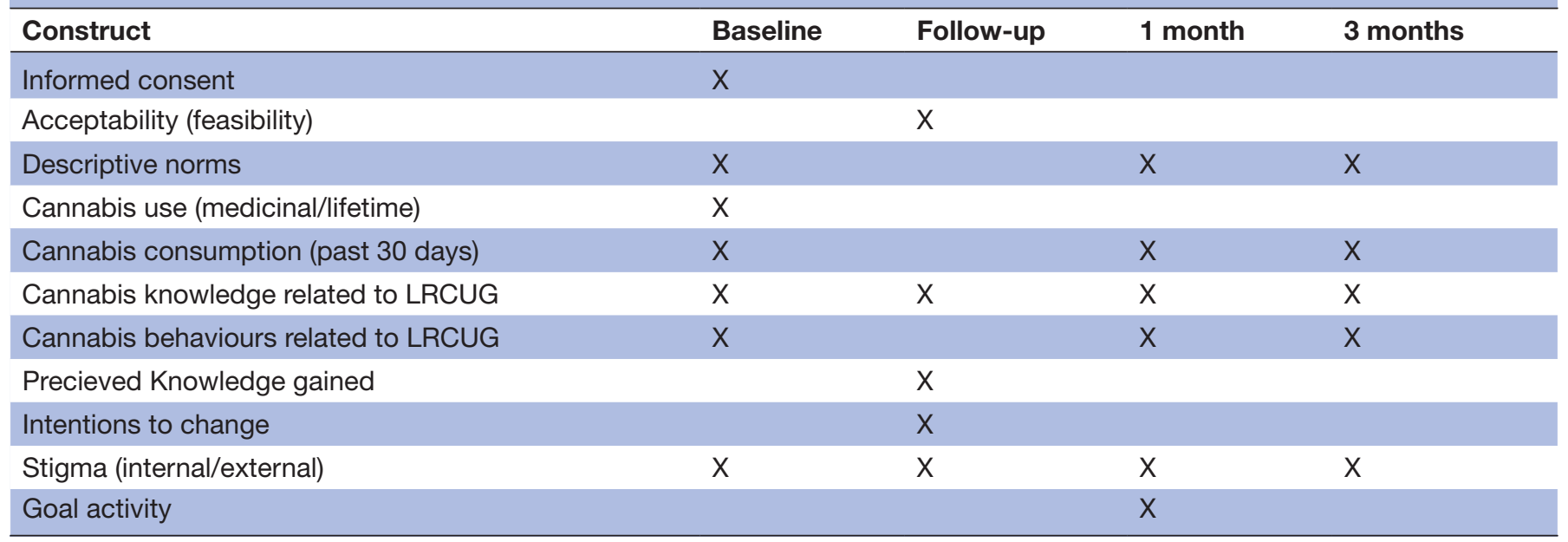

The CUDIT-R is an 8-item screening instrument, where scores of 6 or greater have been previously demonstrated to reflect the likely presence of a CUD among a sample of postsecondary students. ${ }^{39}$ The CUDIT-R was selected as it has been demonstrated to be both a reliable $(\alpha=0.83)$ and concurrently valid measure. ${ }^{39}$

\section{Lower-Risk Cannabis Use Guidelines Knowledge}

Eight items were created for the purpose of objectively measuring participants' cannabis related knowledge as it pertains to the LRCUG. These include: lower-risk methods of administration (eg, edibles vs inhalation of burnt cannabis), lower-risk cannabis products based on cannabidiol (CBD) and tetrahydrocannabinol (THC) concentrations (eg, higher ratios of $\mathrm{CBD}$ and lower amounts of THC) and riskiness of synthetics cannabinoids. Examples of these items include:

1. 'Synthetic cannabis products do not present with additional risks when compared with more natural cannabis products.' Possible responses include strong agree-strongly disagree.

2. 'If you were planning to purchase cannabis for your own personal consumption (OR if you are a non-user and a friend were to ask your advice about which product they should purchase for consumption), please select the THC/CBD concentration that you would most likely purchase/recommend'. Possible responses include: (1) THC 10\%-16\% and CBD 0\%-1\%; (2) THC $20.5 \%-25 \%$ and $\mathrm{CBD}<1 \%$; (3) THC $4 \%-8 \%$ and CBD 8\%-16\%; (4) THC 3.6\%-4.6\% and CBD 5.8\%-7.3\%. (Concentrations were selected based on products available on the legal market.)

Preferences for THC/CBD were selected versus past month product selection, as a large portion of Canadian cannabis consumers are still purchasing from illegal sources. For example, results from the most recent National Cannabis Survey (2019) found that 52\% of 15-24-year olds who reported having consumed cannabis endorsed purchasing some of their cannabis from an illegal supplier. ${ }^{40}$ Thus, we were concerned that a sizeable portion of our participants would not know the CBD/ THC contents of their cannabis and may not be able to give an accurate report.

\section{Behaviours}

A total of five items were developed for the purposes of measuring behaviours related to the LRCUG. These items include whether participants have an immediate biological relative with psychosis or addiction, frequency of consumption over the last 30 days, frequency of deep inhalation techniques, methods of administration, frequency of synthetic use and driving while under the influence. Where applicable, each item is phrased to measure engagement of these behaviours over the past 30 days.

\section{Perceived knowledge gained}

Several subjective measures of knowledge will be administered following the Cannabis Café. For example 'As a result of attending the Cannabis Café, I am more aware of riskier cannabis practices' (strongly agree-strongly disagree).

\section{Intentions to change}

Participants will be asked if they intend to change their behaviours after having attended the Café (eg, as a result of attending Cannabis Café, I intend to change or alter my cannabis practices to be more consistent with lowerrisk use-strongly agree-strong disagree). For those who did not endorse past 30-day use, they will be asked if attending the Cannabis Café reinforced their decision to remain abstinent.

\section{Cannabis stigma}

Changes to both external or internal stigma will be explored using two validated instruments. The first is the Social Distance Scale (SDS) ${ }^{41}$ which consists of seven items where the respondent is asked to indicate how willing or unwilling they would be to entertain specific social situations. Research has shown that the scale can 
be easily modified to measure social distance towards substance using populations. ${ }^{42}$ The SDS has been shown to have good internal consistency $(\alpha=0.80){ }^{42}$ Internal stigma among cannabis users will be measured using the internalised subscale of the Substance Use Stigma Mechanism Scale (SUSMS) ${ }^{43}$ The SUSMS has been shown to have high internal consistency $(\alpha=0.90-0.93)$ and reliability among diverse samples. ${ }^{43}$

\section{Goal setting activity}

Participants will be asked to set a SMART goal to be completed over the next 30 days during the immediate follow-up survey (see online supplementary file). At 1-month follow-up, participants will be asked to indicate if they were able to accomplish their goal (strongly agree to strongly disagree). They will have an opportunity to provide open-ended responses indicating why they were or were not able to accomplish their goal, as well as if they would make any revisions to the goal setting activity.

\section{Fidelity}

A number of measures will be used to ensure the fidelity of the Cannabis Café.

1. The trivia and discussion format will follow a semistructured approach, where specific topics, questions and information will be presented using the MentiMeter platform. Similar to a PowerPoint presentation, key points and information can be specified and presented on each slide. This will ensure that all participants are given the same information at each Cannabis Café, which will be monitored by the principle investigator.

2. Similarly, the principle investigator and a supporting research assistant will complete a brief checklist to ensure that the group facilitator and the discussion leaders are following protocol (please see online supplementary file 1 for more details).

3. MentiMeter records the frequency of responses to each item, allowing us to determine participation rates and exposure to information/norm correction by comparing the number of responses to each trivia item to the number of attendees.

\section{Data analysis}

Descriptive statistics will be calculated to describe the sample. Based on the scale of the outcome variable, appropriate parametric and non-parametric inferential statistics will be calculated including analysis of variances, Kruskal-Wallis test and McNemar's tests. Participants' preresponses/postresponses will be compared with measure changes in knowledge, stigma and consumption of cannabis. Where applicable, analyses will control for demographic variables to determine if programme effects are influenced by gender, sex and age. Cases with missing values (including those cases that were lost to attrition) will be included in the final analyses and when possible, missing values will be imputed. To determine if the missing values are random and can be replaced, Little's ${ }^{44}$
Missing Completely at Random Test will be completed. In addition, cases who dropped out from the study will be compared with those who completed the study on variables measured at baseline to determine if significant differences exist before replacement of missing values. Depending on the nature of the missing data, we may opt for a newer analytical technique that can handle missing values (eg, mixed modelling).

\section{ETHICS AND DISSEMINATION}

Participants' email will be maintained up until 3 months post Cannabis Café, so that surveys can be sent and responses can be linked. This information will be stored in an encrypted and password protected document on a secure server. Participants' email will be removed and replaced with a random identifier after completion of the 3-month follow-up survey. Participants will be made aware during consent that they can withdraw their data at any time up until anonymisation. We recognise that some participants may find answering questions distressing, therefore, after each survey, participants will be presented with a list of relevant resources. As noted earlier, a harm reduction specialist will be present at each Cannabis Café to debrief students who may become distressed.

Given that the Cannabis Café will be facilitated as part of course curriculum and delivered within the classroom, several precautions will be taken to ensure that the participants are clear that the Cannabis Café is part of their course curriculum (as agreed on with the instructor) and that the research (ie, the surveys) is optional and outside of class time. To ensure clarity, the principle investigator on the project will visit the class 1 week prior to the initiative to describe the differences between the Cannabis Café (curriculum) and the evaluation (research) and will explain to the students that involvement is voluntary and will have no effect on their grade. Second, given that both non-participants and participants will be present at the Cannabis Café, the evaluation/research will not be mentioned during the delivery of the initiative. This will help ensure that the confidentiality of participation in the surveys and evaluation of the Cannabis Café are maintained, and that the research project remains separate from course material. Third, given that non-participants will be in attendance, beyond counting the number of attendees and the total number of responses to each trivia item, no research data will be collected during the Cannabis Café. Participants will be made aware of these precautions as part of the informed consent process. Finally, given that the Cannabis Café will be delivered in a group context, participants' confidentiality cannot be guaranteed. However, all students will be encouraged to maintain the confidentiality of their peers.

The dissemination plan will include the development of a Cannabis Café guide to facilitate uptake and replication at other postsecondary campuses. The guide will include a description of each component of the initiative and the delivery protocol. A complementary webinar will 
be facilitated following the development and dissemination of the guideline to provide stakeholders interested in offering the Cannabis Café with additional support. Finally, a manuscript will be prepared and submitted to describe the findings from the study.

\section{DISCUSSION}

The legalisation of cannabis represents an important shift in drug policy in both Canada and in USA. Emerging adults represent a vulnerable group given that their use of cannabis remains high relative to other age groups. Few education programmes to mitigate cannabis related harm have been described and evaluated in the literature. ${ }^{18}$ Thus, evidenced based initiatives in this area are needed. This study will attempt to address this gap by describing and evaluating a novel initiative that addresses cannabis related harm using a brief educational approach among a sample of postsecondary students. This initiative will be informed by evidence, building on components that have been shown to reduce substance related harm among postsecondary students including normative feedback, personal goal setting and the dissemination of strategies to reduce harm. ${ }^{19}$

Of significance is that none of the brief interventions addressing cannabis described in the literature has attempted to reduce stigma. Stigma related to substance use is associated with negative outcomes. ${ }^{31}$ By incorporating components previously demonstrated to reduce stigma related to mental illness and substance use the Cannabis Café may demonstrate benefits that may extend beyond individual behavioural change by reducing negative beliefs towards those who consume cannabis.

If the Cannabis Café is shown to be feasible and the initiative proves useful in modifying cannabis consumption, or if changes are realised in stigma, a more rigorous trial aiming to evaluate the initiative using a random control group design would be warranted. Moreover, if the format of the initiative is shown to be acceptable by students, the Cannabis Café could be repurposed to address other substances commonly used among postsecondary students.

\section{LIMITATIONS}

The study has a number of important limitations. First, several measures were created for the purposes of this study and do not represent psychometrically validated instruments. This included our measures of satisfaction, knowledge uptake and adherence to lower-risk guidelines. As a result, findings from these measures will be interpreted with caution, as their reliability and validity have not been established. Second, given that the Cannabis Café will be delivered in the context of the classroom, participants will attend alongside their fellow classmates. This may limit disclosures of personal experiences including personal use.
Given the exploratory nature of the study and the observational prospective design, no casual conclusions can be drawn. Furthermore, these findings will be limited by a number of confounding factors including changes in policy (eg, we will be recruiting participants during the legalisation of cannabis edibles), which may influence cannabis consumption. Another potential cofounder is when the Cannabis Café is delivered during the school year. It has been previously demonstrated that postsecondary students demonstrate marked fluctuations in their consumption of alcohol throughout the semester. ${ }^{45}$ Similar trends may exist with respect to cannabis consumption and thus, the date when the Cannabis Café is delivered may act as a confounding variable. Finally, the sample will be largely comprised of participants enrolled in courses with a mental health or healthcare focus, limiting the generalisability of the findings. Similarly, findings from the study will not be generalisable to populations beyond postsecondary students. Despite these limitations, the proposed study will serve as foundational work to determine if a comprehensive investigation is warranted.

Cannabis has become a common topic within the public discourse given the recent legalisation of recreational cannabis in Canada and the movement towards legalisation in USA. This shift in law and access offers a unique opportunity for targeted dissemination of information to support university students to make evidenced informed decisions about cannabis use. Part of the justification for legalisation of cannabis in Canada was the government's commitment to specific health objectives including educating Canadians about the potential benefits, risks and harms of cannabis use, along with the prevention of high risk patterns of consumption. ${ }^{46}$ Based on the current dearth of evidenced based harm reduction and education programmes for cannabis, novel methods are needed. The objective of the study is to address this gap by evaluating a psychoeducation programme enhanced by normative feedback and goal setting to address cannabis related stigma and to promote harm reduction among university students through dissemination of LRCUG.

Contributors All authors contributed to the writing of the manuscript and the design of this study. JM was primarily responsible for the development of the Cannabis Café including the evaluation design and preparing the first draft of the manuscript. JMS oversaw the development of the Cannabis Café, assisted in the evaluation design and made significant contributions to the protocol and manuscript. JS assisted in the development of the Cannabis Café, evaluation plan and assisted in the development of the manuscript. DRC provided guidance in the development of the evaluation and provided assistance in preparing the manuscript.

Funding This work was supported by Health Canada, Substance Use and Addictions Program grant number 1819-HQ-000109.

Competing interests None declared.

Patient consent for publication Not required.

Ethics approval The study was approved by the University of Calgary Conjoint Health Research Ethics Board (\#REB18-1364).

Provenance and peer review Not commissioned; externally peer reviewed.

Open access This is an open access article distributed in accordance with the Creative Commons Attribution Non Commercial (CC BY-NC 4.0) license, which permits others to distribute, remix, adapt, build upon this work non-commercially, 
and license their derivative works on different terms, provided the original work is properly cited, appropriate credit is given, any changes made indicated, and the use is non-commercial. See: http://creativecommons.org/licenses/by-nc/4.0/.

\section{ORCID iD}

Joel Mader http://orcid.org/0000-0003-2321-272X

\section{REFERENCES}

1 Canadian Centre on Substance Use and Addiction. Cannabis use, harms, and perceived risks among Canadian students, 2019. Available: https://www.ccsa.ca/sites/default/files/2019-04/CCSACanadian-Students-Cannabis-Harms-Risks-Report-at-a-Glance2019-en.pdf [Accessed Jun 2019].

2 Holmes LM, Popova L, Ling PM. State of transition: marijuana use among young adults in the San Francisco Bay area. Prev Med 2016;90:11-16.

3 Sarvet $\mathrm{AL}$, Wall MM, Keyes KM, et al. Recent rapid decrease in adolescents' perception that marijuana is harmful, but no concurrent increase in use. Drug Alcohol Depend 2018;186:68-74.

4 Miller AM, Rosenman R, Cowan BW. Recreational marijuana Legalization and college student use: early evidence. SSM Popul Health 2017;3:649-57.

5 Government of Canada. National cannabis survey, first quarter 2019 2019. Available: https://www150.statcan.gc.ca/n1/daily-quotidien/ 190502/dq190502a-eng.htm

6 Memedovich KA, Dowsett LE, Spackman E, et al. The adverse health effects and harms related to marijuana use: an overview review. CMAJ Open 2018;6:E339-46.

7 Lopez-Quintero C, Pérez de los Cobos J, Hasin DS, et al. Probability and predictors of transition from first use to dependence on nicotine alcohol, cannabis, and cocaine: results of the National epidemiologic survey on alcohol and related conditions (NESARC). Drug Alcohol Depend 2011;115:120-30.

8 Di Forti M, Morgan C, Dazzan P, et al. High-Potency cannabis and the risk of psychosis. Br J Psychiatry 2009;195:488-91.

9 Gabrys R, Porath A. Clearing the smoke on cannabis: regular use and cognitive functioning, 2019. Available: https://www.ccsa.ca/ sites/default/files/2019-04/CCSA-Cannabis-Use-Cognitive-EffectsReport-2019-en.pdf [Accessed Jun 2019].

10 Arria AM, Caldeira KM, Bugbee BA, et al. The academic consequences of marijuana use during College. Psychol Addict Behav 2015;29:564-75.

11 Suerken CK, Reboussin BA, Egan KL, et al. Marijuana use trajectories and academic outcomes among college students. Drug Alcohol Depend 2016;162:137-45.

12 McKiernan A, Fleming K. Canadian youth perceptions on cannabis. Ottawa, Ont: Canadian Centre on Substance Abuse, 2017. https:// www.ccsa.ca/sites/default/files/2019-04/CCSA-Canadian-YouthPerceptions-on-Cannabis-Report-2017-en.pdf

13 Mader J, Smith JM, Afzal AR, et al. Correlates of lifetime cannabis use and cannabis use severity in a Canadian university sample. Addict Behav 2019;98:106015.

14 Halladay JH, Scherer J, MacKillop J, et al. Brief interventions for cannabis use in emerging adults: a systematic review, meta-analysis, and evidence MAP. Drug Alcohol Depend 2019.

15 Manthey J. Evaluation of the lower risk cannabis use guidelines [thesis] 2013.

16 Fischer B, Russell C, Sabioni $\mathrm{P}$, et al. Lower-risk cannabis use guidelines (LRCUG): an evidence-based update. Am J Public Health 2017;107.

17 Fischer B, Dawe M, McGuire F, et al. Feasibility and impact of brief interventions for frequent cannabis users in Canada. J Subst Abuse Treatment 2013;44:132-8.

18 Jeffries ER, Lemke AW, Shah SM, et al. Addictive behavior interventions among college students. Curr Addict Rep 2016;3:368-77.

19 Scott-Sheldon LA, Carey KB, Elliott JC, et al. Efficacy of alcohol interventions for first-year college students: a meta-analytic review of randomized controlled trials. J Consult Clin Psychol 2014;82:177-88.

20 Riggs NR, Conner BT, Parnes JE, et al. Marijuana eCHECKUPTO go: effects of a personalized feedback plus protective behavioral strategies intervention for heavy marijuana-using college students. Drug Alcohol Depend 2018;190:13-19.

21 American College Health Association. American College Health Association - National College Health Assessment II: Canadian Reference Group Executive Summary Spring 2019. Silver Spring, MD: American College Health Association, 2019. https://www. acha.org/documents/ncha/NCHA-II_SPRING_2019_CANADIAN REFERENCE GROUP EXECUTIVE SUMMARY.pdf
22 Neighbors C, Geisner IM, Lee CM. Perceived marijuana norms and social expectancies among entering college student marijuana users. Psychol Addict Behav 2008;22:433-8.

23 Pearson MR, Hustad JTP, Neighbors C, et al. Personality, marijuana norms, and marijuana outcomes among college students. Addict Behav 2018;76:291-7.

24 Buckner JD. College cannabis use: the unique roles of social norms, motives, and expectancies. J Stud Alcohol Drugs 2013;74:720-6.

25 Hathaway AD, Comeau NC, Erickson PG. Cannabis normalization and stigma: contemporary practices of moral regulation. Criminology Criminal Justice 2011;11:451-69.

26 Health Canada. Canadian cannabis survey 2019, 2019. Available: https://www.canada.ca/en/health-canada/services/publications/ drugs-health-products/canadian-cannabis-survey-2019-summary. html [Accessed Dec 2019].

27 Bottorff JL, Bissell LJ, Balneaves LG, et al. Perceptions of cannabis as a stigmatized medicine: a qualitative descriptive study. Harm Reduct J 2013;10:2.

28 Brown SA. Stigma toward marijuana and heroin users. $J$ Psychoactive Drugs 2015;47:213-20.

29 Palamar JJ. A pilot study examining perceived rejection and secrecy in relation to illicit drug use and associate stigma. Drug Alcohol Rev 2012;31:573-9.

30 Luoma JB, Twohig MP, Waltz T, et al. An investigation of stigma in individuals receiving treatment for substance abuse. Addict Behav 2007;32:1331-46.

31 Global Commission on Drug Policy. The world drug perception problem: Countering prejudices about people who use drugs. Geneva: Switzerland, 2017. http://www.globalcommissionondrugs. org/wp-content/uploads/2018/01/GCDP-Report-2017_PerceptionsENGLISH.pdf

32 Corrigan PW, Larson JE, Rüsch N. Self-stigma and the "why try" effect: impact on life goals and evidence-based practices. World Psychiatry 2009;8:75-81.

33 Wynn Y, Spithoff S, Buchman DZ. Cannabis legislation provides an opportunity to strengthen primary care substance use counseling. Can Fam Physician 2019;65:777-9.

34 Smith JM, Mader J, Szeto ACH, et al. Cannabis use for medicinal purposes among Canadian university students. Can J Psychiatry 2019;65:351-5

35 Elliott JC, Carey KB. Correcting exaggerated marijuana use norms among College abstainers: a preliminary test of a preventive intervention. J Stud Alcohol Drugs 2012;73:976-80.

36 American College Health Association. American College Health Association-National College Health Assessment II: Canadian Reference Group Executive Summary Spring 2016. 2016; Hanover, MD. Available: https://www.acha.org/documents/ncha/NCHAII\%20SPRING\%202016\%20CANADIAN\%20REFERENCE\% 20GROUP\%20EXECUTIVE\%20SUMMARY.pdf [Accessed June 2019].

37 Bowen DJ, Kreuter M, Spring B, et al. How we design feasibility studies. Am J Prev Med 2009;36:452-7.

38 Adamson SJ, Kay-Lambkin FJ, Baker AL, et al. An improved brief measure of cannabis misuse: the cannabis use disorders identification Test-Revised (CUDIT-R). Drug Alcohol Depend 2010;110:137-43.

39 Schultz NR, Bassett DT, Messina BG, et al. Evaluation of the psychometric properties of the cannabis use disorders identification test - revised among college students. Addict Behav 2019;95:11-15.

40 Government of Canada. National cannabis survey, third quarter, 2019, 2019. Available: https://www150.statcan.gc.ca/n1/dailyquotidien/191030/dq191030a-eng.htm

41 Link BG, Cullen FT, Frank J, et al. The social rejection of former mental patients: understanding why labels matter. Am J Sociol 1987:92:1461-500

42 Brown SA. Standardized measures for substance use stigma. Drug Alcohol Depend 2011;116:137-41.

43 Smith LR, Earnshaw VA, Copenhaver MM, et al. Substance use stigma: reliability and validity of a theory-based scale for substanceusing populations. Drug Alcohol Depend 2016;162:32-43.

44 Little RJA. A test of missing completely at random for multivariate data with missing values. J Am Stat Assoc 1988;83:1198-202.

45 Tremblay PF, Graham K, Wells S, et al. When do first-year college students drink most during the academic year? an Internetbased study of daily and Weekly drinking. J Am Coll Health 2010;58:401-11.

46 Health Canada. Canada legalizes and strictly regulates cannabis, 2018. Available: https://www.canada.ca/en/health-canada/news/ 2018/10/canada-legalizes-and-strictly-regulates-cannabis.html [Accessed Jun 2019]. 